\title{
Novel Local Free Energy Minimum on the Cu(001) Surface
}

\author{
Mikhail Ovsyanko, Georgiana Stoian, Herbert Wormeester, ${ }^{*}$ and Bene Poelsema \\ MESA+ Research Institute, University of Twente, P. O. Box 217, 7500 AE Enschede, The Netherlands
}

(Received 15 December 2003; published 17 August 2004)

\begin{abstract}
High-resolution LEED (low-energy electron diffraction) data of $\mathrm{Cu}(001)$ reveal an uniaxial in-plane lattice reconstruction by $1 \%$. One-dimensional nanogrooves induced by ion bombardment involve the creation of steps that enable this reconstruction. This is the first verification of van der Merwe's prediction of step facilitated reconstruction. We confirm the predicted dependence on step orientation: $\langle 100\rangle$ steps allow stress-relief and $\langle 110\rangle$ steps do not, consistent with the known elastic anisotropy. Similar behavior is predicted for other nonreconstructed (001) surfaces of $3 d$ and $4 d$ metals.
\end{abstract}

The creation of a surface requires the breaking of atomic bonds. The resulting lower coordination number makes the surface atoms strive for smaller interatomic distances as noted first by Pauling [1]. This induces in general an inward relaxation of the exposed layer and considerable tensile in-plane stress. Needs [2] calculated the tensile stresses for Al-metal surfaces as high as $\sim 10 \mathrm{GPa}$. Dodson [3] performed thin slab calculations for the (001)-surfaces of $\mathrm{Ni}, \mathrm{Ag}, \mathrm{Cu}, \mathrm{Pd}, \mathrm{Au}$, and Pt. $\mathrm{He}$ obtained a surface strain of several percents, increasing from Ni to Pt. Indeed, it has been found experimentally [4] that some (001)-surfaces of transition and noble metals do reconstruct. Their termination is a (111)-like overlayer that periodically matches the (001)-bulk structure. The decision whether the (001)-surface does reconstruct or not is quite subtle. The adsorption of small amounts of atoms or molecules can lead to deconstruction $[5,6]$. In an ab initio density-functional-theory study, Fiorentini et al. $[7,8]$ investigated the tendency for (001)-transition metal surfaces to reconstruct. They find that surfaces of the metals at the end of the $5 d$ transition series such as Ir, $\mathrm{Pt}$, and $\mathrm{Au}$ reconstruct, whereas their $4 d$ counterparts $\mathrm{Rh}$, $\mathrm{Pd}$, and $\mathrm{Ag}$ do not. Reconstruction requires bond rearrangements, leading to significant energy losses due to the disruption or stretching of bonds between the mismatched layer and that underneath. The reconstruction results from a delicate balance between surface-substrate mismatch and strain related energy gain $[7,8]$. Their high strain energy favors reconstruction for the $5 d$ (001)-surfaces, while it is too small for their $4 d$ and $3 d$ counterparts. The surfaces of the $3 d$ and $4 d$ metals remain unreconstructed in agreement with experimental observations.

The claim by Müller et al.[9] of an in-plane reconstruction of clean $\mathrm{Cu}(001)$ created a sensation. They found a contraction of about $1 \%$ from their LEED (low-energy electron diffraction) $I$-V-analysis. Their finding provided a nice framework for the contraction of $1 \%$ found for the pseudomorphic growth of $\mathrm{Fe} / \mathrm{Cu}(001)$ [10-12]. The $\mathrm{Cu}(001)$ in-plane reconstruction was challenged both experimentally $[13,14]$ and theoretically [15]. Müller et al. [16] conceded and attributed their findings to either a "lower lateral crystallinity" of their surface or "systematic errors affecting the accuracy of their analysis." In a later LEED-I-V-study, Walter et al.[17] indeed obtained smaller in-plane relaxation by introducing an energy dependent inner potential.

We find experimental evidence for a new local minimum in the free energy of $\mathrm{Cu}(001)$. Indeed, $\mathrm{Cu}(001)$ has a strong tendency for an in-plane lattice contraction of $1 \%$. It only prevails on surfaces with a finite amount of steps, when oriented along the $\langle 100\rangle$-azimuth. Note that $\langle 100\rangle$ is the soft direction with respect to deformation [18]. This geometry permits to relieve tensile stress. This result is of general importance to the $3 d$ and $4 d$ (001)-metal surfaces with implications for the creation of nanostructures on or in these surfaces and the structure of hetero-epitaxial islands.

Our method of choice to characterize the morphology of surfaces is spot profile analysis low-energy electron diffraction (SPA-LEED), with a resolution of $\sim 0.1 \%$ of the Brillouin Zone (BZ). All experiments have been conducted in ultrahigh vacuum (pressure $<10^{-10}$ mbar).

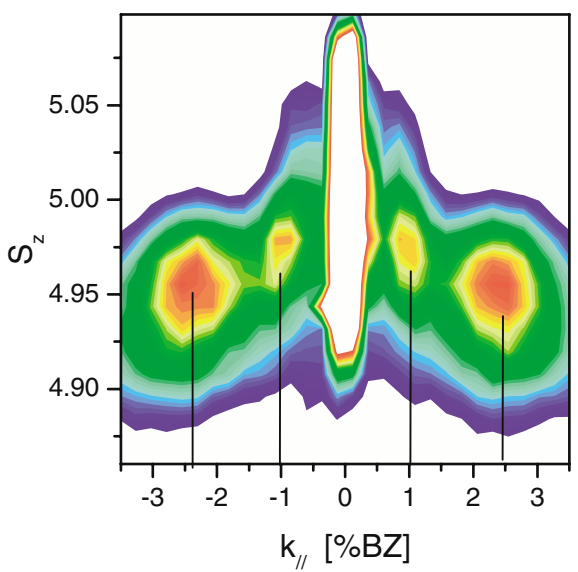

FIG. 1 (color online). Plot of the specular beam profile along the [010] azimuth after $21600 \mathrm{~s}$ sputtering at $235 \mathrm{~K}$ for perpendicular scattering factor $\mathrm{S}_{z}$ between 4.92 and 5.10. Note that the intensity of the $(0,0)$ spot is cut off (shown as white) in order to enhance the diffraction features. 
After a standard cleaning procedure, the flat $\mathrm{Cu}(001)$ surface has a mean terrace width of $80 \mathrm{~nm}$, as derived from the FWHM of the $(0,0)$-beam in SPA-LEED. We have extensively looked, but in accordance with Refs. [13-15], never found any indication for in-plane lattice reconstruction on the clean and flat $\mathrm{Cu}(001)$ surface.

The experiments that led to the observation of an inplane reconstruction started by bombarding the $\mathrm{Cu}(001)$ surface at a selected temperature with $800 \mathrm{eV} \mathrm{Ar}^{+}$ions at a flux of $2 \times 10^{14}$ ions $/ \mathrm{m}^{2} \mathrm{~s}$. The polar angle of incidence is $80^{\circ}$ and the azimuth direction chosen along [100]. Under these conditions highly regular and only two layer deep nanogrooves are formed, running parallel to the plane of incidence of the ions [19]. The distance between the nanogrooves, or better, the periodicity of the onedimensional nanopattern, depends on temperature, ion energy, flux, and fluence. Figure 1 shows typical diffraction features as a function of the parallel wave vector $\mathrm{k}_{/ /}$ in SPA-LEED and the perpendicular scattering factor $S_{z}$ after bombardment with a fluence of about $5 \times$ $10^{18}$ ions $/ \mathrm{m}^{2}$. The scattering factor $\mathrm{S}_{z}$ is defined by $\mathrm{S}_{z}=$ $2 \mathrm{~d}_{001} / \lambda$, where $\mathrm{d}_{001}$ is the spacing between the atom planes normal to (001) and $\lambda$ the electron wavelength. In these data, obtained with the substrate at $235 \mathrm{~K}$, two eye-catching features are visible next to the dominant

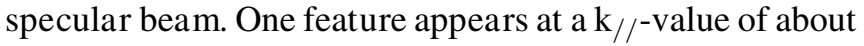
$2.4 \% \mathrm{BZ}$. This feature is related to the periodicity of the nanogroove pattern, corresponding to $102 \pm 6 \AA$. The other feature emerges at about $1 \% \mathrm{BZ}$, i.e., a periodicity of about $250 \AA$. In similar experiments, performed at $200 \mathrm{~K}$ and $175 \mathrm{~K}$, the nanogroove pattern has a periodicity of about $71 \AA$ and $69 \AA$, respectively. In all these cases we observe a feature in the specular beam profile that corresponds to a much larger length scale of $250 \pm 25 \AA$.

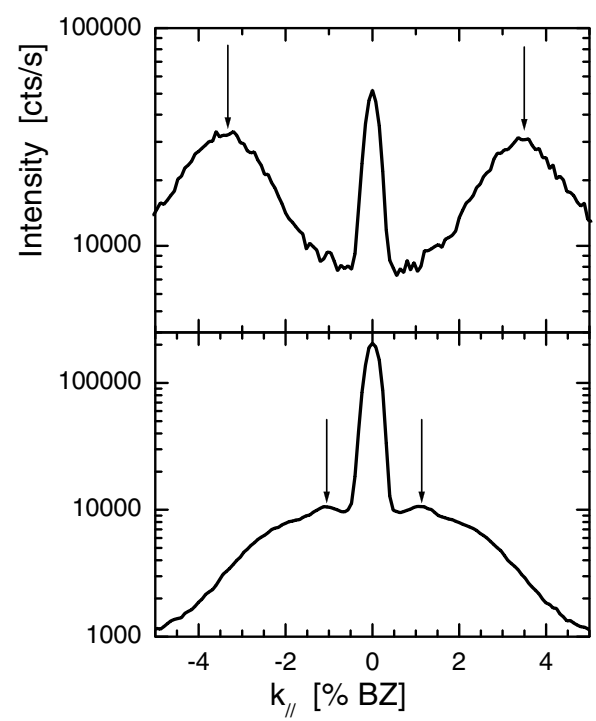

FIG. 2. SPA-LEED profile along [010] after sputtering for $3600 \mathrm{~s}$ at $235 \mathrm{~K}$ (top) and subsequent annealing at $290 \mathrm{~K}$ for $500 \mathrm{~s}$ (bottom).
Within the accuracy, the latter feature coincides with that observed after bombardment at $235 \mathrm{~K}$ (Fig. 1). We emphasize that the persistent feature appears at a fixed,

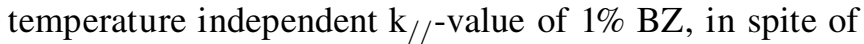
the clear temperature dependence of the nanogroove periodicity.

The specular beam profile obtained in a completely different experiment is shown in Fig. 2. The upper panel shows data measured after bombarding $\mathrm{Cu}(001)$ again at $235 \mathrm{~K}$ (cf. Fig. 1), but now after a six times smaller fluence (bombardment time $3600 \mathrm{~s}$ ). The well-developed wings indicate a nanogroove separation of $75 \AA$. Subsequently the substrate is heated to $290 \mathrm{~K}$. The evolution of the surface morphology is illustrated by the specular beam profile in the lower panel of Fig. 2, obtained after a $500 \mathrm{~s}$ anneal at $290 \mathrm{~K}$. The initially clearly visible wings not only decrease in intensity but also move toward the central peak, indicating an increase of the periodicity of the nanogroove pattern. Also, the intensity of the main peak increases, demonstrating substantial flattening of the surface. We note that again, a small maximum feature beside the main peak at $\Delta \mathrm{k}_{/ /} \sim 1 \% \mathrm{BZ}$ emerges. This feature is persistent and its position does not depend on the annealing time. It remains present even after leaving the sample at $290 \mathrm{~K}$ for as long as $10.000 \mathrm{~s}$. Annealing of the initial structures at $250 \mathrm{~K}$ and at $270 \mathrm{~K}$ leads to a similar appearance of small peaks, corresponding to $250 \AA$.

The experiments described above show a very persistent feature emerging at $\Delta \mathrm{k}_{/ /} \sim 1 \% \mathrm{BZ}$. It corresponds to a length scale of $250 \pm 25 \AA$ along [010], i.e., normal to the nanogrooves. Its appearance is independent of the substantially varying thermal history. The diffraction feature does not change its position even at temperatures with many diffusion processes active on the surface. These combined observations demonstrate a thermodynamic origin of this feature.

The observations indicate an in-plane contraction of the lattice parameter of $1 \%$. This phenomenon agrees with the calculated tensile stress of (001)-faces of $3 d, 4 d$, and $5 d$ metals $[3,7,8]$. The reconstruction results from a delicate balance between surface-substrate mismatch and strain release $[7,8,15]$. We agree and reiterate that this balance does argue against reconstruction for smooth $\mathrm{Cu}(001)$, with $\mathrm{Cu}$ being a $3 d$-metal. However, the actual situation obtained after bombarding the $\mathrm{Cu}(001)$ sample at grazing incidence along [100] deviates from this ideal situation. We do observe satellites along the [010]azimuth but not along [100]. Instead of an isotropic inplane surface plane contraction, the striped pattern only allows uniaxial in-plane contraction normal to the grooves. The easier accommodation of lattice misfits in the presence of atomic steps was predicted theoretically by van der Merwe [20].

We elaborate on the uniaxial in-plane contraction a bit further by considering a one-dimensional model. 
Following van der Merwe [21-23], the periodic first-layer atom-substrate potential is approximated by a truncated Fourier series with an amplitude $W$. The underlying 1D substrate is assumed completely rigid. The lateral interaction in the first layer is modeled with springs, with a constant $\gamma$. The potential energy of a chain of $\mathrm{N}$ atoms, following the notation of Markov [24], given by

$$
E=\frac{\gamma a^{2}}{2} \sum_{n=0}^{N-2}\left(\xi_{n+1}-\xi_{n}-f\right)^{2}+\frac{W}{2} \sum_{n=0}^{N-1}\left[1-\cos \left(2 \pi \xi_{n}\right)\right]
$$

In this, $a$ is the bulk lattice constant, $f=(b-a) / a$ the misfit with $b$ the lattice constant of the unstrained firstlayer, and $\xi_{n}$ the relative position of the $\mathrm{n}^{\text {th }}$ first-layer atom with respect to the minimum of the potential well in units of $a$. The first term accounts for residual strain due to the different spacing between the first-layer atoms and $b$. The last term reflects the energy cost due to the misfit between the first layer and the substrate. The full energy minimum results then in a variation of the parallel lattice constant for the first-layer atoms. In this case, the chain runs in the [010] azimuth. The energy $W$ mimics the energy difference between a $\mathrm{Cu}$ atom in the fourfold hollow site and in the on-top position. We estimate that $W=0.8 \mathrm{eV}$, about twice the activation energy for diffusion over the bridge site [25]. The strain relief energy has been calculated using first principles by Fiorentini et al. $[7,8,15]$. For $\mathrm{Cu}(001)$, uniaxially contracted by $1 \%$, the energy gain amounts to $1.12 \mathrm{eV}$. This consideration does not incorporate the energy for the creation of dislocations, necessary to let the smooth surface reconstruct $[7,8]$. This dislocation energy clearly outweighs the strain relief energy, assuring that no reconstruction of the intact surface is observed [15]. However, the starting situation in our experiments is not the smooth but rather the nanogrooved surface. First we may consider the resulting surface as a two-level system. In all situations of relevance here, the distance between the nanogrooves is smaller than 100 atomic spacings. The upper level can now relax without a need for the incorporation of additional dislocations. Obviously, the lower level remains unreconstructed. The system can now fully profit from the energy gain predicted by the Frenkel-Kontorova expression and the numbers quoted above: the presence of atomic steps on the surface allows local in-plane contraction. This holds even more if, as in our case, the steps alternate from step-down to step-up. The atoms on the higher of the two levels will lock in around the minimum energy positions and the unfavorable positions are not occupied. We assume first that both exposed levels occupy $50 \%$ of the surface and that the periodicity of the step-up step-down sequence equals $250 \AA$. Then the upper level allows an in-plane contraction at a much lower cost, as compared to the smooth surface: the "expensive" positions remain now unoccupied.
The morphology of the real nanogrooved surface is quite complex, even within a simple two-level model in which only the upper level is reconstructed. To obtain insight in the resulting beam profile we have simulated two basic cases with different characteristics. The 1D Frenkel-Kontorova model (Eq. (1)) predicts that relaxation of the upper level layer results in atomic positions that deviate laterally from the bulk lattice and undergo a corresponding variation in height. The latter is called corrugation and its amplitude amounts to a maximum of $a \sqrt{2}$. To mimic the effect of corrugation we have calculated the diffraction profile obtained from a chain of atoms with a periodicity of $100 a$, their lateral positions on bulk lattice positions and the vertical positions varying as a cosine with amplitude $a \sqrt{2}$. The chain is positioned on a 1D bulk crystal. The diffraction has been calculated using the kinematic approximation and an electron mean free path of $0.8 \mathrm{~nm}$. This results in strong symmetric features at $1 \%$, with a FWHM corresponding to the instrumental resolution, see Fig. 3 (dashed line). In accordance with the Frenkel-Kontorova model (Eq. (1)), also the lateral position of the first-layer atoms in general deviate from the bulk position. This dispersion in lateral position was also simulated within the same framework (solid line). The dispersion in lateral position leads to a strongly reduced feature compared to the intensity of the $(0,0)$ beam. This intensity reduction can simply be explained by the fact that the effective height distribution of the atoms is narrower. Additional calculations for various fractions occupied by the upper level (coverage), varying from 10 to $50 \%$, show a small but persistent spectral density at $1 \%$ of the first Brillouin Zone. This spectral density remains rather constant with coverage. The latter can be understood easily: for "low" coverage the atoms can fully relax and still remain quite near to the bottom of the potential wells. The effective upper layer lattice

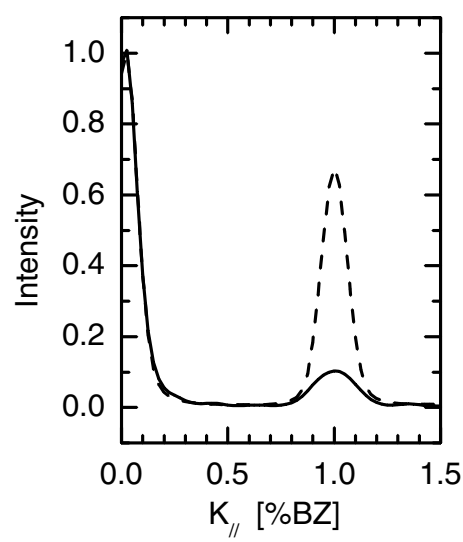

FIG. 3. Simulated diffraction intensity at $S_{z}=4.97$ of an atomic chain that only shows a height variation (dashed line) and an additional variation in lateral interatomic distance according to the Frenkel-Kontorova model (solid line). The calculated results have been convoluted with the instrumental response function. 
distance is then close to $0.99 a$. For "high" coverage the situation approaches the result for the second calculation described above. In the latter case, the density at $1 \% \mathrm{BZ}$ is mainly caused by the intrinsic length scale of $100 a$ of the corrugation profile. Even with this simple model the obtained strength is of the order of the experimental data.

We note that Stepanyuk et al. $[26,27]$ have calculated in-plane (and out-of-plane) relaxation of lattice parameters near the edges of two-dimensional close packed adatom islands. In-plane contractions in the order of also $1 \%$ have been obtained, the actual value being dependent on the size of the islands. We note that our stable diffraction feature at $\sim 1 \% \mathrm{BZ}$ has a distinctly different origin. Neither its position nor its magnitude do depend on the size of the structures.

van der Merwe and Kunert [28] also predicted that relief of the misfit energy facilitated by atomic steps does depend on their orientation. We also confirm this result experimentally. We observe the feature at $\Delta \mathrm{k}_{/ /}=$ $1 \% \mathrm{BZ}$ only after bombarding the surface along $\langle 100\rangle$. Bombarding along the $\langle 110\rangle$ azimuth never leads to additional diffraction features. Relieving tensile stress is thus facilitated by preexisting $\langle 100\rangle$ and not by $\langle 110\rangle$ oriented steps. This observation is rationalized in terms of anisotropic elastic properties of the $\mathrm{Cu}(001)$ surface. Calculations by Ozolins et al.[18] show that $\langle 100\rangle$ is the soft direction with regard to lattice deformation. In contrast, the $\langle 111\rangle$ and the $\langle 110\rangle$ directions are hard directions.

From the width of the diffraction patterns along the grooves, we estimate the average length of the grooves at several hundred $\AA$. Reconstruction parallel to the grooves would thus involve accommodation of a much higher mismatch energy. We also note that relaxation of tensile stress normal to the grooves poses a local minimum of the free energy. A relatively mild anneal at $400 \mathrm{~K}$ is sufficient to overcome the energy barrier separating the local minimum from the global free energy minimum leading to extended smooth (001) terraces.

In conclusion, we have observed an in-plane lattice parameter that is $1 \%$ smaller than the bulk lattice parameter. This is due to the relief of the tensile stress inherent to (001)-surfaces of metals. The reconstruction, not observed for macroscopic (001)-terraces, is enabled by the presence of $\langle 100\rangle$ oriented atomic steps and not by $\langle 110\rangle$-steps. This is attributed to anisotropic resistance against elastic deformations. The local free energy minimum, associated with the in-plane lattice contraction, is separated from the global one, leading to unreconstructed $\mathrm{Cu}(001)$ surface, by a relatively low-energy barrier, which can be overcome by a mild anneal at $400 \mathrm{~K}$. Our results reconcile quite recent controversies in literature. They are considered important for the delicate interplay between mismatch and tensile stress energies on fcc(001) metal surfaces in general.
This work is part of the research program of the Stichting Fundamenteel Onderzoek der Materie (FOM), financially supported by the Nederlandse Organisatie voor Wetenschappelijk Onderzoek (NWO).

*Electronic address: Corresponding author: h.wormeester@utwente.nl

[1] L. Pauling, The Nature of the Chemical Bond (Cornwell University, Ithaca, 1960).

[2] R. J. Needs, Phys. Rev. Lett. 58, 53 (1987).

[3] B.W. Dodson, Phys. Rev. Lett. 60, 2288 (1988).

[4] K. Heinz, G. Schmidt, L. Hammer, and K. Müller, Phys. Rev. B 32, 6214 (1985).

[5] D. G. Fedak and N. J. Gjostein, Phys. Rev. Lett. 16, 171 (1966).

[6] C. Romainczyk, J. R. Manso, K. Kern, K. Kuhnke, R. David, P. Zeppenfeld, and G. Comsa, Surf. Sci. 336, 362 (1995).

[7] V. Fiorentini, M. Methfessel, and M. Scheffler, Phys. Rev. Lett. 71, 1051 (1993).

[8] V. Fiorentini, M. Methfessel, and M. Scheffler, Phys. Rev. Lett. 81, 2184 (1998).

[9] S. Müller, M. Kottcke, R. Metzler, P. Bayer, L. Hammer, and K. Heinz, Phys. Rev. Lett. 75, 2859 (1995).

[10] P. Bayer, S. Müller, P. Schmailzl, and K. Heinz, Phys. Rev. B 48, 17611 (1993).

[11] S. Müller, P. Bayer, A. Kinne, P. Schmailzl, and K. Heinz, Surf. Sci. 322, 21 (1995).

[12] H. Magnan, D. Chanderis, B. Vilette, O. Hechmann, and J. Lecante, Phys. Rev. Lett. 67, 859 (1991).

[13] A. P. Baddorf, A. K. Swan, and J. F. Wendelken, Phys. Rev. Lett. 76, 3658 (1996).

[14] K. Robinson, W. Moritz, F. Jona, and M. A. van Hove, Phys. Rev. Lett. 76, 3659 (1996).

[15] S. Oppo and V. Fiorentini, Phys. Rev. Lett. 81, 4278 (1998).

[16] S. Müller, M. Kottcke, L. Hammer, and K. Heinz, Phys. Rev. Lett. 76, 3660 (1996).

[17] S. Walter, V. Blum, L. Hammer, S. Müller, K. Heinz, and M. Giesen, Surf. Sci. 458, 155 (2000).

[18] V. Ozolins, C. Wolverton, and A. Zunger, Appl. Phys. Lett. 72, 427 (1998).

[19] S. Van Dijken, D. de Bruin, and B. Poelsema, Phys. Rev. Lett. 86, 4608 (2001).

[20] J. H. van der Merwe, Phys. Rev. B 37, 2892 (1988).

[21] F. C. Frank and J. H. van der Merwe, Proc. R. Soc. London A 198, 205 (1949).

[22] F. C. Frank and J. H. van der Merwe, Proc. R. Soc. London A 198, 216 (1949).

[23] J. H. van der Merwe, Philos. Mag. A 45, 145 (1982).

[24] I.V. Markov, Crystal Growth for Beginners (World Scientific, Singapore, 1995).

[25] H. Dürr and J. F. Wendelken, Surf. Sci. 328, L527 (1995).

[26] O.V. Lysenko, V.S. Stepanyuk, W. Hegert, and J. Kirschner, Phys. Rev. Lett. 89, 126102 (2002).

[27] V.S. Stepanyuk, D. I. Bazhanov, W. Hergert, and J. Kirschner, Phys. Rev. B 63, 153406 (2001).

[28] J. H. van der Merwe and H. Kunert, Phys. Rev. B 37, 2902 (1988). 